\title{
POST-TRAUMATIC STRESS DISORDER IN AN ADOLESCENT WITH ASPERGER SYNDROME
}

\author{
I-Cheng Lin ${ }^{1}$, Li-Yang Tseng ${ }^{1} \&$ Yao-Tung Lee ${ }^{1,2,3}$ \\ ${ }^{I}$ Department of Psychiatry, Shuang Ho Hospital, Taipei Medical University, New Taipei City, Taiwan \\ ${ }^{2}$ Department of Psychiatry, School of Medicine, College of Medicine, Taipei Medical University, Taipei, Taiwan \\ ${ }^{3}$ Center of Dementia, Shuang Ho Hospital, Taipei Medical University, New Taipei City, Taiwan
}

received: 19.4.2020;

revised: 22.5.2020;

accepted: 3.6 .2020

$* * * * *$

\section{INTRODUCTION}

Post-traumatic stress disorder (PTSD) is relatively common but often unrecognized in primary care settings. Being a disabling psychiatric disorder, identifying and treating PTSD may be important. Additionally, it had been reported that persons with autistic traits may be at elevated risk of PTSD symptoms after the trauma (Roberts et al. 2015). Being one of the autism spectrum disorders, Asperger Syndrome (AS) thus may have higher PTSD risk subsequent to trauma. Here, we report one case to highlight clinical wisdom. Informed consent from this case report had been received.

\section{CASE PRESENTATION}

A 16-year-old high school girl visited the psychiatric clinic for being difficult in concentration and in falling asleep. Besides, nightmares, depression and angry easily outbursts were also mentioned. A detailed interview was performed and revealed that she performed well academically from elementary to junior high. However, there started the problem of social interaction since the final year of elementary school which she contributed to her lake of interests in common topics such as popular idols and TV series. Thereafter, she became isolated from social groups and got a nickname of "test machine" from her classmates. One month before visiting the clinic, she was hit severely by her father after an intense argument resulting in minor brain concussions and retinal edema. After that, she suffered from several PTSD symptoms including re-occurring image of her father's domestic violence, nightmares, persistent anger and horror toward her father, and having difficulty in concentration and in falling asleep. There also existed the behavior of selftalking to criticize her as useless and she started to find various excuses to escape from the school. Besides, the angry outburst increased remarkably accompanied with more and more severe verbal and behavioral violence. She was then brought to the clinic for help.

\section{RESULTS}

The relevant physical examination, laboratory testing and imaging showed no specific abnormality. In the psychological assessment, she achieved extraordinary results on the Wechsler Adult Intelligence ScaleIII but the scores showed a significant gap between the verbal and performance ability (VIQ=144, PIQ=119). During the evaluation, we noted her persistent preoccupation with parts of objects contributing to overlook the generality.

At the same time, she was also noted of impairments in nonverbal behaviors which made her difficult in social interaction and failure to develop peer relationships. Besides, she presented with a fairly straightforward and apparently inflexible thinking process that she rarely hid or disguised her feelings and thoughts. She was highly suspected to be a case of Asperger syndrome.

In the following time, she was treated with antidepressant (sertraline, $25 \mathrm{mg} /$ day) and hypnotics (lorazepam, $0.5 \mathrm{mg}$ before sleep) daily. At the same time, cognitive behavioral therapy was also arranged. The status of anxiety, sleep quality and nightmare improved significantly after 2 months of treatment. According to her mother, her hostility toward father also decreased gradually. In the following half year, no further episode had happened.

AS is a neuro-developmental defect characterized by impairments in emotion regulation, social interaction and communication. It had been suggested that the effects of trauma will be greater for those who has difficulty in emotion regulation and inter-personal interactions (Cloitre et al. 2005). making AS a higher risk of developing PTSD. Besides, biological evidences had reported amygdala dysfunction to be a critical feature of AS (Kleinhans et al. 2009) while amygdala and its functional interactions with the ventromedial prefrontal cortex had been proposed to be associated with PTSD (Stevens et al. 2013), suggesting the connection between AS and PTSD. However, literature about the comorbidity of AS and PTSD is still limited. 
Our opinion is that, the post-traumatic responses of AS should get more attention. In our case, the treatment included not only medications but also the psychotherapy. We suggest that the identification of PTSD in the AS and the correlated therapeutic strategy may be an issue worth investigation.

\section{References}

1. Cloitre M, Miranda R, Stovall-McClough KC, Han H: Beyond PTSD: Emotion regulation and interpersonal problems as predictors of functional impairment in survivors of childhood abuse. Behavior Therapy 2005; 36:119-124

2. Kleinhans NM, Richards T, Weaver KE, Liang O, Dawson $G$, Aylward E: Brief report: biochemical correlates of clinical impairment in high functioning autism and Asperger's disorder. J Autism Dev Disord 2009; 39:1079-1086

3. Roberts AL, Koenen KC, Lyall K, Robinson EB, Weisskopf MG: Association of autistic traits in adulthood with childhood abuse, interpersonal victimization, and posttraumatic stress. Child Abuse Negl 2015; 45:135-142

4. Stevens JS, Jovanovic T, Fani N, Ely TD, Glover EM, Bradley B, Ressler KJ: Disrupted amygdala-prefrontal functional connectivity in civilian women with posttraumatic stress disorder. J Psychiatr Res 2013; 47:1469-1478

Acknowledgements: None.

Conflict of interest: None to declare.

Correspondence:

Yao-Tung Lee, MD

Department of Psychiatry, Shuang Ho Hospital, Taipei Medical University No. 291, Zhongzheng Road, Zhonghe District, New Taipei City 235, Taiwan E-mail:11685@s.tmu.edu.tw 